To be presented at the 1998 IEEE World Congress on Computational Intelligence in Anchorage, Alaska, May 4-9, 1998

$$
\text { CONF-980538-- }
$$

\title{
Reinforcement Function Design and Bias for Efficient Learning in Mobile Robots*
}

\author{
Claude F. Touzet \\ Computer Science and Mathematics Division \\ Oak Ridge National Laboratory \\ P.O. Box 2008, Bldg. 6012 \\ Oak Ridge, TN 37831-6367 \\ Juan Miguel Santos \\ Department Computación \\ University of Buenos Aires \\ Buenos Aires, Argentina

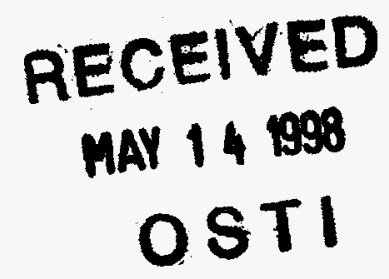

\begin{abstract}
"This submitted manuscript has been authored by a contractor of the U.S. Government under Contract No. DE-AC05-96OR22464. Accordingly, the U.S. Govemment retains a nonexclusive, royalty-free license to publish or reproduce the published form of this contribution, or allow others to do so, for U.S. Government purposes."
\end{abstract}

RECEIVED

JUN 251998

DISTRIBUTION OF THIS DOCUMENT IS UNLMITED

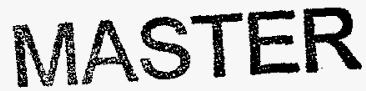

OSTI

\footnotetext{
*Research supported in part by the Office of Basic Energy Sciences, Office of Energy Research, U.S. Department of Energy, under contract No. DE-AC05-96OR22464 with Lockheed Martin Energy Research Corporation; and, portions of this work were also performed during a previous appointment at DIAM-IUSPIM, University of Aix-Marseille III, France.
} 


\section{DISCLAIMER}

This report was prepared as an account of work sponsored by an agency of the United States Government. Neither the United States Government nor any agency thereof, nor any of their employees, makes any warranty, express or implied, or assumes any legal liability or responsibility for the accuracy, completeness, or usefulness of any information, apparatus, product, or process disclosed, or represents that its use would not infringe privately owned rights. Reference herein to any specific commercial product, process, or service by trade name, trademark, manufacturer, or otherwise does not necessarily constitute or imply its endorsement, recommendation, or favoring by the United States Government or any agency thereof. The views and opinions of authors expressed herein do not necessarily state or reflect those of the United States Government or any agency thereof. 


\section{DISCLAIMER}

Portions of this document may be illegible electronic image products. Images are produced from the best available original document. 


\title{
Reinforcement Function Design and Bias for Efficient Learning in Mobile Robots
}

\author{
Claude Touzet, CESAR, Oak Ridge National Laboratory, TN, USA (touzetc@mars.epm.ornl.gov) \\ Juan Miguel Santos, Dept. Computación, Univ. of Buenos Aires, Argentina (jmsantos@zorzal.dc.uba.ar)
}

\begin{abstract}
The main paradigm in sub-symbolic learning robot domain is the reinforcement learning method. Various techniques have been developed to deal with the memorization/generalization problem, demonstrating the superior ability of artificial neural network implementations. In this paper, we address the issue of designing the reinforcement so as to optimize the exploration part of the learning. We also present and summarize works relative to the use of bias intended to achieve the effective synthesis of the desired behavior. Demonstrative experiments involving a self-organizing map implementation of the Q-learning and real mobile robots (Nomad 200 and Khepera) in a task of obstacle avoidance behavior synthesis are described.
\end{abstract}

\section{Introduction}

Most of the research conducted today for learning in autonomous robots deals with the behavior-based paradigm. This bottom-up approach concentrates on physical systems situated in the world and promotes simple associative learning between sensing and acting. The grounding problem of symbols is yet unsolved [1], so symbolic methods cannot be used (at least alone); and, the burden of learning in robotics falls on sub-symbolic approaches (it is assumed that learning in mobile robotics is aimed at avoiding the need (for the human operator) to model all of the complexities, interactions, or other influences in the world [2]). Among the sub-symbolic learning approaches currently available, reinforcement learning (RL) is one of the most investigated approaches. Real world problems present the challenge of huge problem spaces. Thus, the process of applying robot learning requires addressing the generalization problem. Artificial neural networks implementations (e.g., multilayer perceptron, recurrent networks, self-organizing map) provide today a very powerful tool.

However, it is very difficult to solve most realworld problems with tabula rasa learning techniques. We review in this paper several biases that allow effective learning solutions along with reinforcementfunction (RF) design. Bias is inclusion of initial knowledge such as (a) provision of an external supervisory module, (b) pre- programmed reflexes, (c) instance-based learning, and (d) using a trainer.

\section{A behavior}

In the early $80 \mathrm{~s}, \mathrm{R}$. Brooks [1], along with others, advocated a bottom-up approach, concentrating on physical systems situated in the world autonomously carrying out tasks of various sorts. The behavior-based approach promotes simple reflex-like actions and associative learning between sensing and acting. The architecture of the system (e.g., robot) is decomposed into task-achieving modules, also called behaviors. A learning robot is a robot that can improve its behavior or the encoding of behavior as a result of interaction with the real world environment [3]. In this canonical model, as shown in Fig. 1, situations in the world are perceived; and, an action or a mental attitude is generated using a situationaction mapping function.

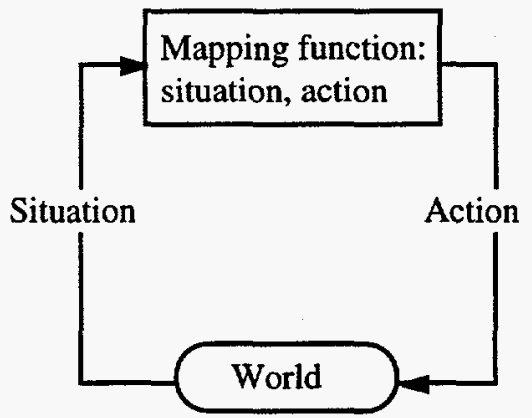

Fig. 1. A behavior is a mapping function between situations of the world as sensed by the sensors and actions undertaken by the actuators. Internal states may help disambiguate the situations.

\section{Reinforcement function}

$R L$ dates back to the early days of cybernetics and work in statistics, psychology, neuroscience and computer science. In the last five-to-ten years, it has attracted rapidly increasing interest in the machine learning and artificial intelligence communities. Its promise is beguiling - a way of programming robots by reward and punishment without needing to specify how the task (i.e., behavior) is to be achieved [4]. RL synthesizes a mapping function 
between situations and actions by maximizing the reinforcement signal (Fig. 2). It promotes learning of relevant associations. In RL, the behavior is synthesized by using, as a unique source of information, a scalar ${ }^{1}$--the so-called reinforcement value, which evaluates behavior actions: The robot receives either positive or negative reinforcements according to the utility (i.e., desirability) of the situation entered as a consequence of the performed action. There is no separation between a learning phase and a utilization (or test) phase. RL allows one, at least in principle, to bypass the problems of building an explicit model of the behavior to be synthesized or a meaningful learning base needed for supervised learning [5].

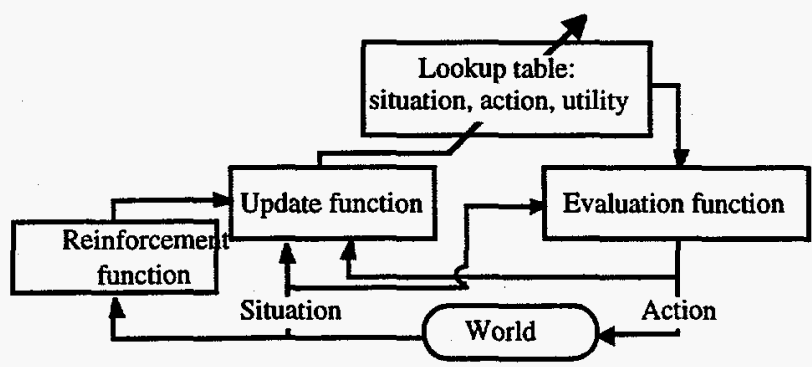

Fig. 2. Reinforcement learning architecture.

However, any difficulty in the use of the learning paradigms is the result of a situation space being so large that, combined with all possible actions, an exhaustive exploration or memorization of all situation-action pairs is impossible. This fact nullifies the impact of the proof of asymptotic convergence [6] which requires a discrete coding of the situation-action pairs (tabular representation) and an infinite number of repetitions of every action for every situation.

\section{Endowing the robot with generalization capabilities}

A solution to the incredible large size of the situation-action space is the generalization process: The use of experienced situation-action pairs to deal with new unknown situations. Therefore, researchers have proposed implementations of the different learning paradigms [7][8][9] that allow generalization. Artificial neural networks are among the most effective implementation tools currently known for learning to interpret complex real world sensor data [10]. Several neural networks models are at the disposition of the researcher that have demonstrate enhanced performances[11]. Figure 3 shows a comparison between the neural network implementations (CMLP and Q-KOHON) of the Qlearning [12] has been done [13]. The self-organizing map

1 A scalar is used in the simplest form of RL. However, it is our opinion that a too-complex RF which provides a specific value for each entered situation, does not respect the primary interest of the RL paradigm; i. e., the possibility to avoid a modelization of the searcher behavior.
Q-learning implementation requires less memory and learns faster than all the others. The increase in performance (learning time) is superior to 40 when compared to the basic Q-learning implementation (left).

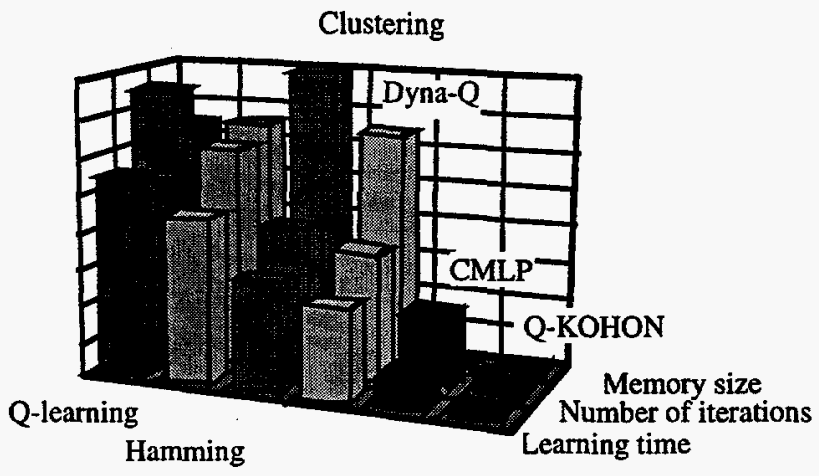

Fig. 3. Comparison of several implementations of Q-learning on a task of learning an obstacle avoidance behavior.

Even if, as it has been shown, efficient RL implementations are available, the main difficulty is to teach the right thing; that is to make the robot converge precisely to the desired behavior. There are two problems: convergence (of the learning) and convergence to the right behavior.

\section{Reinforcement function design}

RFs are usually hand-tuned and emerge after lots of experiments. Convergence of the learning depends on the quality of the exploration. Too few non-null received rewards, and convergence will never occur. Too many received rewards, and the synthesized behavior may be too general to be of any use. The number of received non-null rewards during the (random part of the) exploration depends only on the form of the RF. We propose the following particular definition of RF, which has only one threshold parameter $(\theta+$ or $\theta-)$ per (positive or negative) reward:

$$
R F\left(s_{1}, \ldots, s_{u}\right)=\left\{\begin{array}{l}
+1 \text { if } g_{1}\left(s_{1}, \ldots, s_{u}\right)>\theta_{+} \\
-1 \text { if } g_{2}\left(s_{1}, \ldots, s_{u}\right)<\theta_{-} \\
0 \quad \text { otherwise }
\end{array}\right.
$$

where $\left(s_{1}, \ldots, s_{u}\right)$ is the output readings of the sensors, $g_{1}()$ and $g_{2}()$ are any functions linking the sensor data to the rewards.

An Update Parameter Algorithm (UPA) [14] has been developed to adjust automatically during the learning phase the threshold values: $\theta+$ and $\theta-$. UPA optimizes, in this case, the exploration part of the learning phase by achieving and maintaining pre-defined ratios ${ }^{2}$ of positive

\footnotetext{
2 If there is no positive reward, the evaluation function built during the
} 
and negative rewards. UPA tuned parameters are tested using a mobile Nomad 200 robot on a task of synthesis of an obstacle avoidance behavior. The performance of the learning is evaluated by plotting the distance to the obstacles. A Q-KOHON [15] implementation is used involving 16 neurons, and a neighborhood of 4 and 18 inputs (16 IR sensor values, 1 action value, $1 Q$ value). The self-organizing map is used in the following way: The best action to undertake in a world situation is given by the neuron that has the minimal distance to the input situation and to a $Q$ value of value +1 (Fig. 4a). The selected neuron corresponds to a triplet (situation, action, $Q$ value). It is this particular action which should offer the best reward in the world situation (Fig. 4b).

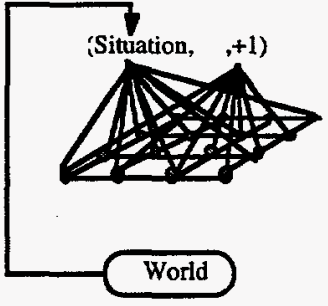

(a)

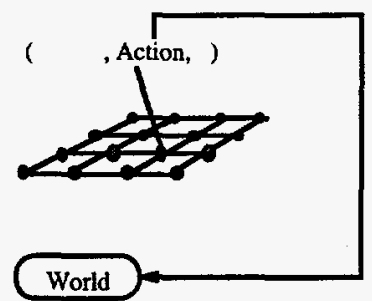

(b)
Fig. 4. Selection of the best action to perform in the world situation. The Kohonen map is used as an associative memory: information is probed with part of it. (a) The world situation and a $Q$ value of +1 are given as inputs. (b) The answer is a selected neuron which weights give situation, $Q$ value and the associated action.

The learning algorithm updates the $Q$ value weight and, also, the situation and action weights. The neuron corresponding to the situation and the action effectively performed is selected. The distance used here is different from the one used during the exploration process. It includes the situation and action vectors but nothing concerning the $Q$ value. Together with the selected neuron, the neighbors are also updated. During the learning, the influence on the neighbors decreases inversely proportional to the number of iterations. The properties (local representation of the probability densities) of the self-organizing map allow us to predict that, if a correct behavior is learned (i.e., only positive rewards are experienced), then all neurons will code positive $Q$ values [13].

Figure 5 presents several experiments that have been made with threshold values, both close to or far from those obtained with the UPA. The only combination allowing a distance to the obstacles greater than a random move selection behavior corresponds to the threshold configuration given by the UPA. This fact shows the

learning phase will have " 0 " as maximum value and the policy cannot select effective actions. If there is no negative reward, the robot can remain in a dead-end situation forever. If there is no null reward, the evaluation function will be non-continuous at the frontier between positive and negative situation-action pairs. validity of the threshold parameters obtained, suggesting that further experiments are required to measure the impact of these first steps in RF design.

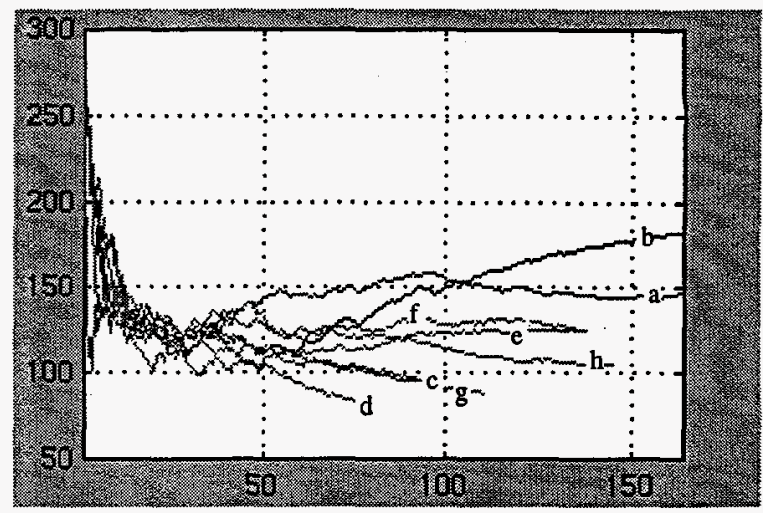

Fig. 5. Distance to the obstacles with respect to the number of obstacles encountered during the 300 experiment iterations (200 learning plus 100 test):

(a) is obtained by a random move selection behavior (no learning is involved),

(b) is the learned behavior corresponding to the values given by UPA $(\theta+=390, \theta-=1150)$,
(c) $(\theta+=195, \theta-=2300)$, (d) $(\theta+=780, \theta=575)$,
(e) $(\theta+=195, \theta=1150)$, (f) $(\theta+=780, \theta=1150)$,
(g) $(\theta+=390, \theta-=2300)$, (h) $(\theta+=390, \theta=575)$.

\section{Necessary biases to incorporate}

There is a variety of RL techniques that work effectively on a variety of small problems, but very few of these techniques scale well to larger problems. This comes from the fact that it is very difficult to solve highly complex problems with tabula rasa learning techniques. Biases must be incorporated to allow effective learning solutions.

\subsection{Addition of external modules dealing with sequences of actions}

The RL algorithm task is to improve the cumulative reward over time. Despite a good learning phase (i.e., only positive rewards experienced after the initial exploration phase), the obtained behavior does not always exhibit the expected behavior. For example, in our experiments of learning an obstacle avoidance behavior with the miniature mobile robot Khepera [16], we observe, for the synthesized behaviors, a large distribution of covered distances. Figure 6 displays four curves corresponding to four different experiments. A behavior may exhibit a predilection for forward moving, backward moving, small movements, or for change in its policy during the experiment. There is also another behavior which is perhaps the most rewarding considering our $\mathrm{RF}^{3}$.

\footnotetext{
${ }^{3}$ The robot receives the following reinforcement signals for avoiding: +1 if it is avoiding, or

-1 if a collision occurs, or

0 otherwise.
} 
It is: move forward to an obstacle, stop before the sum of the sensor values is superior to the threshold (2.90), then move backward, and do it all again. This sequence of actions maximizes rewards, but it is something different from the expected obstacle avoidance behavior.

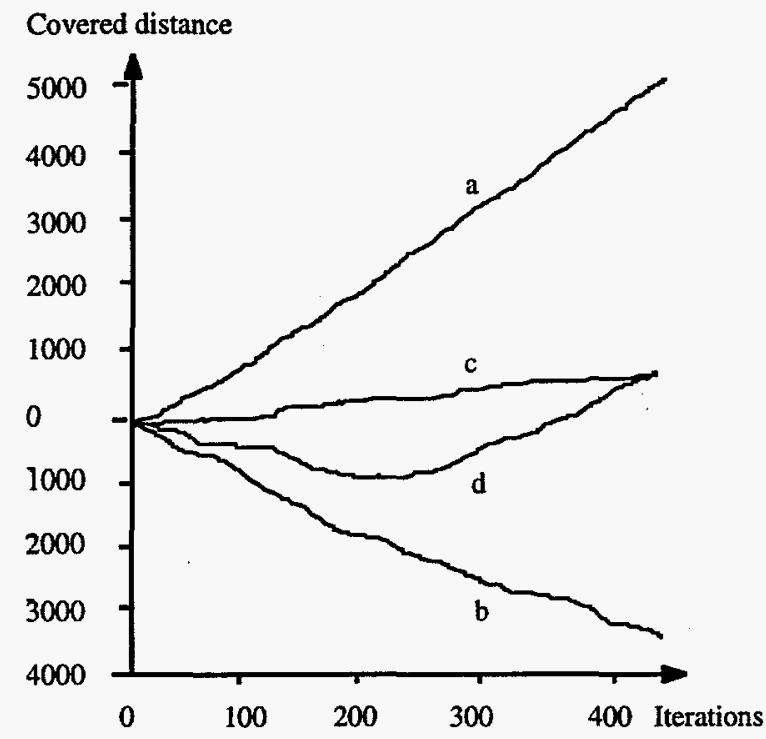

Fig. 6. Distances covered by Khepera during four different experiments of learning an obstacle avoidance behavior. Behavior (a) displays a predilection for forward moving, (b) prefers backward moving, (c) prefers small forward movements, (d) changes its policy at the end of the learning phase (200 iterations).

The learning can be improved with the use of a set of forbidden sequences of actions. Here are, for the three mentioned problems, the corresponding sequences of actions to forbid:

\section{1/ Moving back and forth $\rightarrow$ Alternated} sequences of actions having the same absolute values.

$2 /$ Small movements $\rightarrow>$ long sequences of actions having small absolute values.

3/ Backward avoidance $\rightarrow$ Long sequences of actions having negative values.

All these sequences modify the exploration function. The effect is to suppress the eligibility of actions in a given situation (and a given historical context). On the self-organizing map implementation, the second

The robot is avoiding when the present sum of sensor values is smaller than the last one, the difference being superior to 0.06 . A collision occurs when the sum of the six front sensor values is superior to 2.90 , or the sum of the two back sensor values is superior to 1.95 . closest neuron is selected instead of the first. Experiments point out that only forward behaviors were learned (fig. 7).

Covered distance

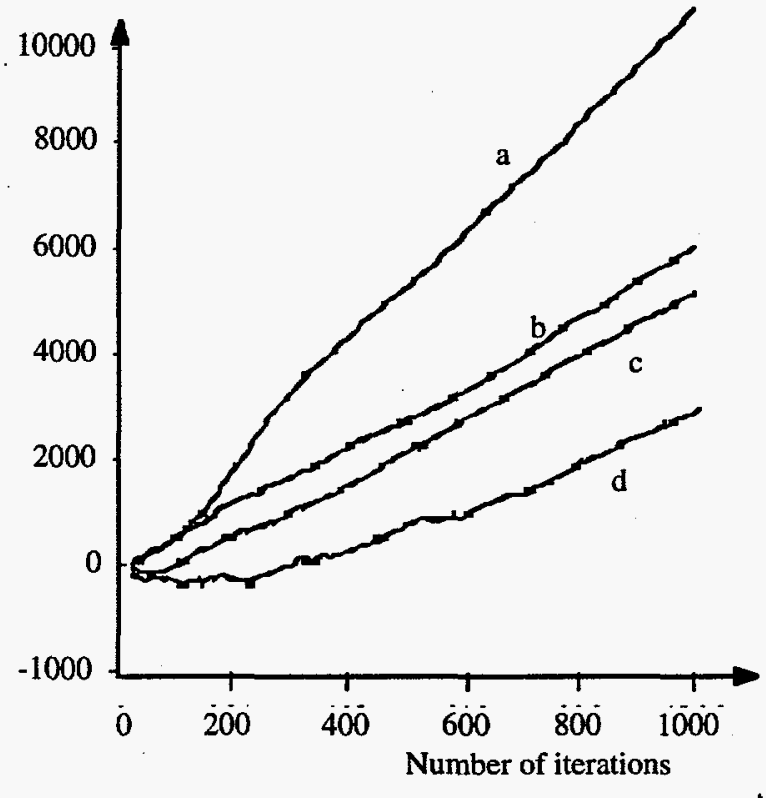

Fig. 7. Covered distances of four different experiments ( $a, b, c, d)$. They differ only through their initial random conditions.

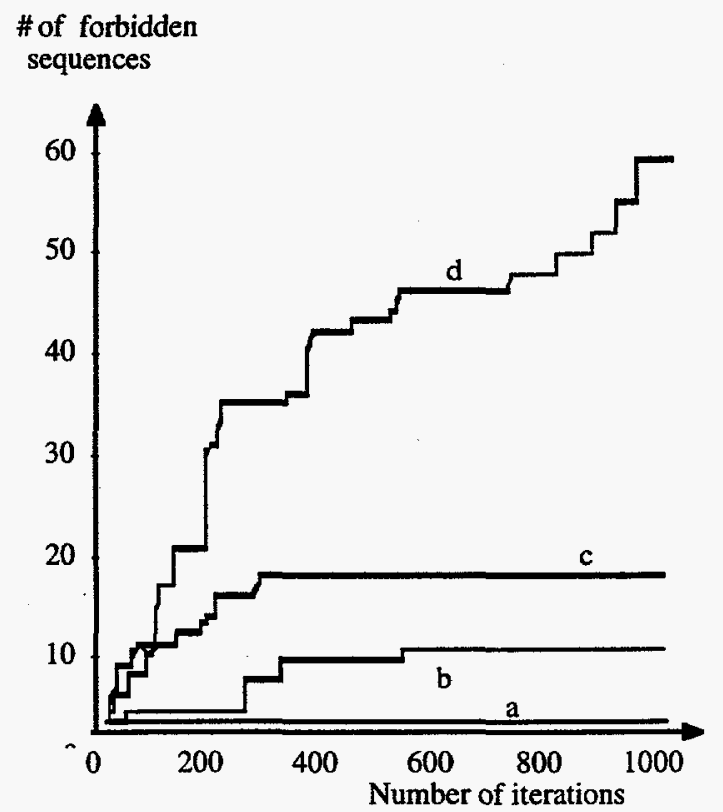

Fig. 8. Number of forbidden sequences used per experiment.

The number of the forbidden sequences used per experiment is reported on Fig. 8. It shows that not all experiments need a large implication of this module. A 
too-quick analysis would state that the involvement of the forbidden sequence module is proportional to the inverse of the covered distance. In fact, the learned behavior starts from initial random conditions. The less the behavior favorites long-covered distances, the more the involvement of the forbidden sequence module. This is particularly visible in experiment (d).

\subsection{Pre-programmed reflexes}

Millán [17] provides the robot with a set of reflexes; i.e., initial knowledge that will allow a better exploration of the search space (Fig. 9). This initial knowledge can be ameliorated through learning. A set of basic reflexes is used every time the connectionist controller fails to generalize correctly its previous experience to the current situation; i.e., the evaluation function does not find an input neuron matching the current situation. The connectionist controller associates the selected reflex with the situation in one step. The sensory situation is represented by a new unit of the network, and the selected reflex or situation-action pair is coded into the network weights. This new association is tuned subsequently through RL. The neural network gets control more often as the robot explores the environment.

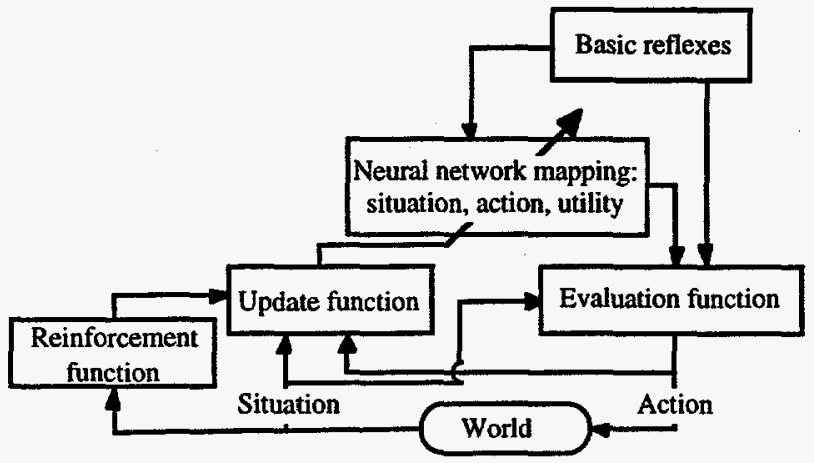

Fig. 9. Learning from basic reflexes.

\subsection{Instance-based learning}

With the same objective as the precedent proposal, instance-based learning [18] can be used to provide the robot with some initial useful information [19]. A sampling of the environment is done prior to any behavior synthesis using a random action generator. Situationaction pairs are recorded in a lookup table (Fig. 10). These information can afterwardsbe used through a user-defined policy. During the following learning phase, the usefulness of this initial knowledge decreases in response to the improved knowledge gained through the learning.

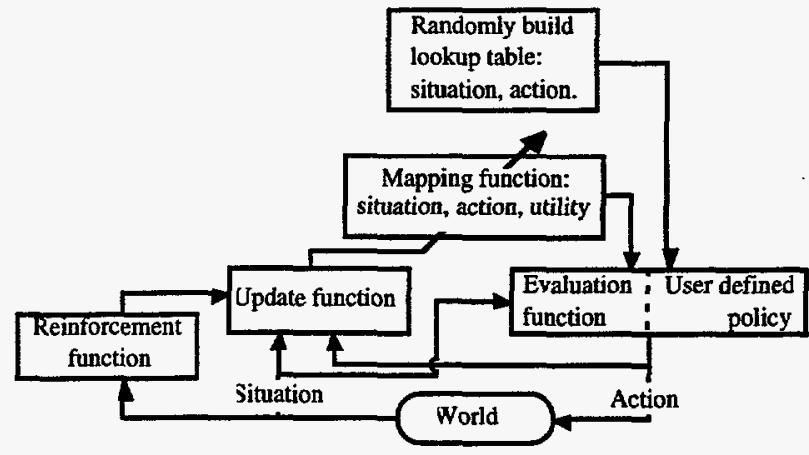

Fig. 10. Instance-based learning used as initial knowledge.

\subsection{Using a trainer}

Dorigo et al. [20] propose to add to the $\mathrm{RL}$ architecture an explicit trainer (Fig. 11) in charge of providing step-by-step guidance by means of immediate reinforcements. The trainer, providing immediate reinforcements, removes the delayed reinforcement problem. The trainer (i.e., the RF) disposes of its own sensors. It is necessary to specify and implement the RF with its own sensors. It could be difficult to imagine having at our disposition important information, the $R F$ sensors, and not giving the data to the robot.

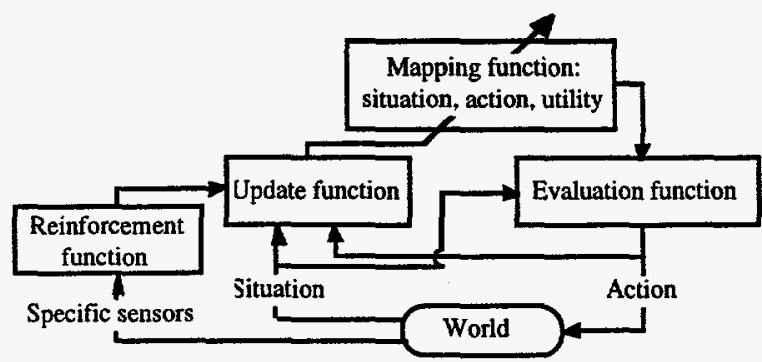

Fig. 11. RL architecture using an explicit trainer.

\subsection{Others bias}

Many other biases have been proposed to help/speed RL application developments. This includes (but is not restricted to):

Shaping [20]: Start with very simple tasks, then increase the difficulty. This method is particularly useful with supervised learning approaches.

Local reinforcement signals [21]: Whenever possible, give local reinforcement signals, rewarding local actions (not far from immediate reinforcement signals). Imitation: Learn by watching another robot. The problem is to get the first robot to perform the task. Today, this first robot must be human controlled. 


\section{Conclusion}

The main paradigm in the sub-symbolic learning robot domain is the $R L$ method. Various techniques have been developed to deal with the memorization / generalization problem which demonstrate the superior ability of artificial neural network implementations. We have addressed here the issue of designing the RF so as to optimize the exploration part of the learning. We have also presented and summarized works relative to the use of bias intended to achieve the effectivesynthesis of the desired behavior.

Based on a particular form of the RF, the Update Parameter Algorithm (UPA) allows the computation during a pure exploration phase of an estimation of the $R F$ threshold values. The test of the tuned parameters using a mobile Nomad 200 robot on a task of synthesis of an obstacle avoidance behavior showed the validity of the $R F$ parameters obtained, suggesting that further experiments are certainly needed to really measure the impact of these first steps in RF design. The UPA use has been restricted here to a pure (without learning) exploration phase. It would certainly be interesting to be able to use the UPA during the learning phase as well. However, the nonuniform distribution of the rewards during the learning phase imposes modifications of the algorithm previously described. It is our desire to address this issue in the near future.

Another attempt has been made to help the combinational implementation of Q-learning to deal with time delay. The use of forbidden sequences of actions provides another way to act on the synthesized behavior without tuning the RF. Experiments with the real miniature robot Khepera in a task of learning an obstacle avoidance confirm the potentialities associated with this approach.

\section{Acknowledgments}

This research was funded in part by the Office of Energy Research, Basic Energy Sciences, of the U.S. Department of Energy, under contract No. DE-AC05960R22464 with Lockheed Martin Energy Research Corporation (C. Touzet). Portions of this work were also performed during a previous appointment (C. Touzet) at DIAM-IUSPIM, University of Aix-Marseille III, France.

\section{References}

[1] R. Brooks, "Intelligence without reason," IJCAI'91, Sydney, 1991.

[2] H. Hexmoor, L. Meeden, and R. Murphy, "Is Robot Learning a New Subfield?", AI magazine, 1997.

[3] Introduction to the Special Issue on Learning Autonomous Robots, M. Dorigo Guest Editor, IEEE Transactions on Systems, Man and Cybernetics - part B, Vol. 26, No. 3, 361-364, June 1996.
[4] L. Kaelbling, M. Littman and A. Moore, "Reinforcement Learning: A Survey," Journal of Artificial Intelligence Research 4, 237-285, 1996.

[5] Sandip Sen and Claude Touzet, "Agent Learning," Invited presentation, First Conf. on Autonomous Agents, Monterey del Rey, CA, 1997

[6] P. Dayan and T. Sejnowski, "TD(1) convergence with probability 1," Machine Learning, 14(3), 1994.

[7] S. Mahadevan \& J. Connell, "Automatic Programming of Behavior-based Robots using Reinforcement Learning," Artificial Intelligence, 55, 2, pp. 311-365, July 1991.

[8] R.S. Sutton, "Reinforcement Learning Architectures for Animats," Proc. of the First Int. Conf. on Simulation of Adaptive Behavior, From Animals to Animats, Edited by J-A Meyer and S.W. Wilson, MIT Press, 288-296, 1991.

[9] A. W. Moore and C. G. Atkeson, Prioritized sweeping: Reinforcement learning with less data and less real time, Machine Learning, 13, 1993.

[10] T. Mitchell, Machine Learning, McGraw Hill, March 1997.

[11] L-J. Lin, "Reinforcement Learning for Robots Using Neural Networks," Ph.D. thesis, Carnegie Mellon University, Pittsburgh, CMU-CS-93-103, January 1993.

[12] C. J. C. H. Watkins, "Learning from Delayed Rewards," Ph.D. thesis, King's College, Cambridge, England, 1989.

[13] C. Touzet, "Neural Reinforcement Learning for Behaviour Synthesis," Special issue on Learning Robot: the New Wave, N. Sharkey Guest Editor, Robotics and Autonomous Systems, 650, 251-281, 1997.

[14] J. M. Santos and C. Touzet, "Automatic Tuning of the Reinforcement Function," Fourth Int. Conf. on Neural Networks and their Applications (NEURAP'98), Marseilles, France, March 1998.

[15] S. Sehad and C. Touzet, "Reinforcement Learning and Neural Reinforcement Learning," ESANN 94, Brussels, April 1994.

[16] F. Mondada, E. Franzi \& P. Ienne, "Mobile Robot Miniaturisation: A Tool for Investigation in Control Algorithms," Third International Symposium on Experimental Robotics, Kyoto, Japan, October 1993.

[17] J. del R. Millán, "Rapid, Safe and Incremental Learning of Navigation Strategies," Special Issue on Learning Autonomous Robots, M. Dorigo Guest Editor, IEEE Trans. on Systems, Man and Cybernetics - part B, Vol. 26, No. 3, 408-420, June 1996.

[18] D. Aha Ed., Lazy Learning, Kluwer, 1997.

[19] J. W. Sheppard and S. L. Salzberg, "A Teaching Strategy for Memory-Based Control," Lazy Learning, D. Aha Ed. , Kluwer, 343-370, 1997.

[20] M. Dorigo and M. Colombetti, Robot Shaping: Developing situated agents through learning, Artificial Intelligence, 71, 2, 321-370, 1994.

[21] M. J. Mataric, "Reinforcement Learning in MultiRobot Domain," Autonomous Robots 4, 73-83, 1997. 